\title{
Tamanho das Empresas, Interação com Universidades e Inovação*
}

\author{
Luciano Martins Costa Póvoa ${ }^{1}$ \\ Sandro Eduardo Monsueto ${ }^{2}$
}

Resumo: Este artigo apresenta um exercício econométrico relacionado à hipótese schumpeteriana de que a tendência a inovar cresce com o tamanho da empresa para um conjunto específico de empresas que interagem com universidades e institutos de pesquisa. Os resultados sugerem que empresas grandes tendem a ser mais inovadoras que as micro e pequenas empresas quando se trata de inovações de produto. Entretanto, essa vantagem de tamanho não se verifica quando são tratadas inovações de processo.

Palavras-chave: empresa; inovação; interação universidade-empresa.

\section{Firm Size, Interaction with Universities, and Innovation}

\begin{abstract}
This paper tests the schumpeterian hypothesis that innovation increases with firm size. The test is performed for a specific set of firms that interact with universities and research institutes. The results suggest that big firms tend to be more innovative in products than small ones. However, this advantage of firm size is not observed for process innovation.
\end{abstract}

Key-words: firm size; innovation; university-firm interaction.

JEL: L25.

\footnotetext{
* Os autores agradem os comentários de Leandro Almeida e Marcelo Pinho. Eventuais erros remanescentes são de nossa inteira responsabilidade.

1 Doutor em Economia pelo Centro de Desenvolvimento e Planejamento Regional (Cedeplar) da Universidade Federal de Minas Gerais (UFMG). Professor do Departamento de Economia da Universidade de Brasília (UnB). E-mail: lpovoa@unb.br

2 Doutor em Economia pela Universidad Autónoma de Madrid (Espanha). Professor do Departamento de Economia da Universidade Federal de Goiás (UFG). E-mail: monsueto@face.ufg.br
} 


\section{Introdução}

Na última década foram lançadas várias políticas que tiveram entre seus objetivos a promoção de uma maior interação entre empresas e universidades (ex. Lei de Inovação e Fundos Setoriais). Tais políticas apóiam-se na ideia de que as empresas raramente inovam sozinhas e de que as universidades e institutos de pesquisa podem contribuir para o processo de inovação colaborando para a geração de projetos inovativos ou para a conclusão de projetos iniciados pelas empresas.

Outro tipo de política nesse período teve como objetivo o aprimoramento da capacidade de inovação das micro e pequenas empresas (MPE, daqui em diante), como o Programa de Apoio à Pesquisa a Micro e Pequenas Empresas.

Os dados da Pesquisa de Inovação Tecnológica (PINTEC) 2008 sugerem que quanto maior o porte da empresa (em termos de pessoal ocupado), maior é a propensão a inovar. A taxa de inovação (produto e/ou processo) foi de 37\%, para empresas de 10 a 99 empregados, e alcançou $72 \%$ para empresas acima de 500 empregados. Tais resultados remetem à antiga hipótese schumpeteriana de que a inovação tende a crescer com o tamanho da firma. Além disso, a importância dada às universidades e institutos de pesquisa como fontes externas de informação para as atividades inovativas também tende a ser maior entre grandes empresas.

O objetivo deste artigo é testar a hipótese schumpeteriana para um conjunto de empresas que interagem com universidades e institutos de pesquisa com base nos dados do BR Survey. Ou seja, entre as empresas que interagem, o tamanho continua sendo importante para diferenciar a propensão a inovar? Caso seja, em que circunstâncias?

Compreender mais a fundo a relação entre tamanho da firma e atividades inovativas é crucial para a elaboração de políticas com o objetivo de subsidiar o P\&D privado. O subsídio deve ser maior para empresas menores? É possível delimitar faixas ótimas de tamanho das empresas que tornaria o subsídio mais eficaz? Embora o presente artigo não trate diretamente dessas questões, pela limitação dos dados, os resultados sugerem que as MPE realizam esforços para inovar significativamente maiores que os das médias e grandes empresas. Esses resultados indicam a necessidade de se aprimorar os estudos nessa área.

A maior limitação deste estudo é não possuir um grupo de controle, pois a amostra contém apenas empresas que interagem com universidades e institutos de pesquisa. Assim, apresentamos um estudo dessa base de dados que poderá ser comparado aos resultados de análises posteriores baseadas nas informações da PINTEC. 
O artigo está estruturado em mais cinco seções, além dessa introdução. A seção seguinte apresenta uma breve revisão da literatura sobre a hipótese schumpeteriana. A terceira seção traz considerações sobre a base de dados. Os resultados das análises descritivas e dos modelos econométricos estão presentes na quarta seção. As conclusões são apresentadas na quinta seção.

\section{Tamanho da empresa e inovação: algumas evidências empíricas}

Em seu livro Capitalismo, Socialismo e Democracia de 1942, Joseph Schumpeter sugeriu, sobretudo nos capítulos 7 e 8, que empresas maiores tendem a ser mais inovadoras e que a inovação está relacionada a mercados mais concentrados, distanciando-se do ambiente de competição perfeita. Tais observações geraram uma onda de artigos nas décadas seguintes com o propósito de testar essas "hipóteses schumpeterianas". Cohen e Levin (1989, p. 1060) sumarizam tais hipóteses debatidas na literatura como (i) a inovação cresce mais que proporcionalmente com o tamanho da empresa e (ii) a inovação cresce com a concentração de mercado.

Os autores reconhecem que tais hipóteses, embora não representem fielmente o pensamento de Schumpeter, levaram vários pesquisadores a desviar da questão central levantada pelo autor; a do entendimento das causas e conseqüências econômicas do avanço tecnológico. Nelson (2006), por exemplo, apresenta uma crítica das interpretações feitas na literatura que induziram às chamadas hipóteses schumpeterianas. Para o autor, Schumpeter realmente observou que, no decorrer do século XX, as etapas de invenção e inovação estavam deixando de ser separadas. A grande empresa passava a ser o local tanto da criação, em seus laboratórios de P\&D, quanto da estratégia de introdução das invenções nos mercados. Entretanto, não se pode depreender que Schumpeter tenha dito que quanto maior a empresa, mais ela inova. A relação entre inovação e tamanho de empresa (bem como estrutura de mercado) é bem mais complexa do que as hipóteses schumpeterianas parecem sugerir.

Em uma ampla resenha da literatura, Cohen e Levin (1989) sugerem que os estudos empíricos haviam sido inconclusivos justamente por não levarem em conta as fontes fundamentais da variação do comportamento inovativo e da performance das firmas e indústrias. Uma análise mais profunda deveria avaliar a estrutura de demanda, as oportunidades tecnológicas (natureza e abundância) e as condições de apropriabilidade dos ganhos dos investimentos em P\&D presentes em cada indústria. 
Além da deficiência em analisar as fontes fundamentais da atividade inovativa, os estudos empíricos destacados pelos autores enfrentaram uma série de dificuldades metodológicas, como por exemplo, limitação dos dados, equações mal especificadas e dificuldades de medidas de input e output da atividade inovativa. A maior parte dos estudos usou alguma medida de input do processo de inovação como variável dependente, tais como gastos em P\&D ou pessoal empregado em atividades de P\&D. Já as medidas de output mais usadas foram o número de patentes. Contudo, esses estudos não levaram em consideração o fato de que patentes só são utilizadas como a principal forma de apropriação dos ganhos de $\mathrm{P} \& \mathrm{D}$ em poucas indústrias, como a farmacêutica (Levin et al, 1987).

Tais esclarecimentos ajudam a entender porque os estudos empíricos sobre a relação entre tamanho da firma e inovação chegam a resultados tão diversos, levando a uma falta de conclusão geral sobre a validade das hipóteses schumpeterianas.

Contudo, os avanços na qualidade dos dados sobre a atividade inovativa permitiram novas avaliações da hipótese de que a propensão a inovar está relacionada ao tamanho da firma. Acs e Audretsch (1987) utilizaram os dados do U.S. Small Business Administration a respeito do número de inovações por firmas, em vez de patentes. Os autores testaram sob quais circunstâncias grandes empresas tem maior vantagem inovativa relativa sobre às pequenas empresas. A principal conclusão do estudo foi relativizar a importância do tamanho das firmas. Os resultados indicaram que grandes empresas tendem a apresentar maiores vantagens inovativas em indústrias intensivas em capital, concentradas e intensivas em propaganda. Já as vantagens inovativas para pequenas empresas tendem a ocorrer em indústrias que estão nos estágios iniciais do ciclo de vida do produto.

O fato de a importância do tamanho da firma poder variar entre as indústrias também é destacado por Freeman e Soete (2008). Os autores indicam que em alguns setores industriais as empresas menores tendem a levar vantagem, como por exemplo, indústrias em que o desenvolvimento tecnológico demanda flexibilidade das atividades de P\&D e agilidade nas tomadas de decisões.

Os estudos mais recentes tem utilizado os dados de pesquisas elaboradas estritamente para captar a inovação nas empresas, tais como Community Innovation Survey (CIS). Uma resenha dos estudos que utilizaram os dados do CIS é apresentada por Mairesse e Mohnen (2010), que apontam para a existência de uma relação positiva entre tamanho da firma e propensão a inovar como um resultado geral. Além disso, os esforços em P\&D (medido geralmente como proporção da receita com vendas) aparecem como a variável significante mais citada nos estudos sobre os determinantes da inovação. Os autores também apontam estudos que indicam que firmas que cooperam (com outras firmas ou com universidades) tendem a gastar mais em P\&D. 
Entre os estudos sobre o caso brasileiro antes de surgir a PINTEC destacamos o de Macedo e Albuquerque (1999), que analisaram a relação entre esforços em P\&D e tamanho da empresa com base nas informações do estudo da Competitividade da Indústria Brasileira. Os autores calcularam as elasticidades do dispêndio em P\&D em relação ao tamanho da empresa, medido pelo seu faturamento. Os resultados sugerem que o esforço em P\&D está desproporcionalmente concentrado em empresas menores. Ao avaliar a relação entre tamanho das firmas e o número de patentes, os autores encontram uma relação em U, o que significa que há uma concentração grande de patentes em empresas menores.

Com a disponibilização dos dados da PINTEC foi possível aprimorar os estudos sobre os determinantes da inovação na indústria brasileira. Kanebley et al (2004), a partir de um estudo não-paramétrico, analisam as características das empresas inovadoras empregando dados da PINTEC. Segundo os autores, entre as principais características que afetam a probabilidade de uma firma ser inovadora estão a orientação exportadora (contínua e ocasional) e o seu tamanho (número de empregados). Além disso, a probabilidade de ser inovadora aumenta mais que proporcionalmente com o tamanho da firma. A variável tamanho da firma também está relacionada à inovação em praticamente todos os setores industriais analisados pelos autores.

Entretanto, mesmo os trabalhos recentes não conseguem lidar com uma das maiores limitações na análise da relação entre tamanho da firma e inovação: a dificuldade (principalmente pela falta de dados) de se avaliar a causalidade inversa, ou seja, o efeito da inovação sobre o crescimento da firma e, conseqüentemente, sobre o seu tamanho (Acs e Audretsch, 1987; Cohen e Levin,1989).

\section{Base de dados}

O presente artigo é limitado em seus objetivos pela natureza dos dados em questão. Contudo, as informações analisadas a seguir são extremamente valiosas para o entendimento da relação entre universidades, institutos de pesquisa e empresas inovadoras.

Para a construção da base de dados foram enviados questionários para as empresas que declararam ter interagido com os grupos de pesquisa no censo do Diretório de Grupos de Pesquisa do CNPq. Um total de 1.688 questionários foi enviado entre abril a novembro de 2009, obtendo uma taxa de resposta de 19,3\% (325 empresas). Ao retirar os questionários respondidos de modo incompleto, as empresas que indicaram investimento em P\&D superior a 30\% da receita nos últimos três anos e as que declararam que 100\% dos empregados 
estão envolvidos em atividades de P\&D restaram 296 empresas ${ }^{1}$.

No questionário foi perguntado a cada empresa se ela havia introduzido alguma inovação nos últimos três anos. No presente estudo, consideramos a firma como inovadora de produto caso tenha introduzido algum produto novo para o país ou para o mundo (o mesmo para inovadoras de processo). Ou seja, essa definição de empresa inovadora é mais rigorosa do que a adotada pela PINTEC, que considera inovadora também a empresa que realizou um aperfeiçoamento substancial de um produto (ou processo) já existente, ou se introduziu um produto considerado apenas pela própria empresa como um produto (ou processo) novo ou substancialmente aperfeiçoado.

As empresas foram classificadas em três grupos de acordo com o tamanho em número de empregados: (i) micro e pequenas empresas (MPE daqui em diante): de o a 99 empregados; (ii) empresa média: de 100 a 499 empregados; e (iii) empresa grande: acima de 500 empregados. A classificação da atividade econômica das empresas foi de acordo com a Classificação Nacional de Avaliação Econômica (CNAE) 2.0.

\section{Resultados}

Nesta seção apresentamos os resultados gerais da taxa de inovação das empresas por tamanho, bem como os resultados do modelo econométrico elaborado para testar a hipótese schumpeteriana entre tamanho e propensão a inovar na amostra em questão.

\subsection{Análise descritiva}

Entre as 296 empresas que compõem a amostra, 101 (34\% do total) são MPE, 94 (32\%) são médias empresas e 101 (34\%) são grandes empresas. $\mathrm{Na}$ amostra total, $58 \%$ das empresas inovaram no período considerado (em produto e/ou processo). Os dados da PINTEC 2008 apontam para uma taxa de inovação de 38,6\%. Dado que nossa definição de inovação é mais restrita que a da PINTEC, esses resultados sugerem que as firmas que interagem com universidades e institutos de pesquisa tendem a ser mais inovadoras do que o conjunto da economia brasileira.

1 Empresas que não responderam o número de empregados e nem se realizaram ou não inovações nos últimos três anos foram retiradas da amostra. Quanto à intensidade dos gastos em $\mathrm{P} \& \mathrm{D}$, exceto empresas de setores de alta tecnologia, raramente uma empresa investe uma média acima de $5 \%$ de sua receita em atividades de P\&D. Realizamos um corte arbitrário em 30\% para tentar captar empresas startups ou pequenas empresas de base tecnológica em fase de maturação. 
A taxa de inovação de produto foi de $51 \%$, enquanto a de processo foi de $32 \%$. Além disso, apenas $6,7 \%$ das empresas implementaram apenas inovação de processo, enquanto $26,7 \%$ implementaram apenas inovação de produto. Ou seja, não só inovação de produto é mais freqüente, como é difícil uma empresa inovadora em produto não ser também inovadora em processo.

A Figura 1 mostra que independente da categoria de tamanho da empresa, a taxa de inovação em produto é superior à de inovação em processo, embora as grandes empresas apareçam como sendo mais inovadoras tanto em produto quanto em processo. Já o padrão de inovação entre MPE e médias empresas na amostra é bastante similar. Esses resultados diferem do verificado para a economia como um todo. Conforme citado na introdução, os dados de inovação da PINTEC 2008 mostram que a taxa de inovação tende a crescer monotonicamente com o tamanho da empresa.

FIGURA 1. BRASIL: TAXA DE INOVAÇÃO DE PRODUTO E PROCESSO POR TAMANHO DA EMPRESA - 2009

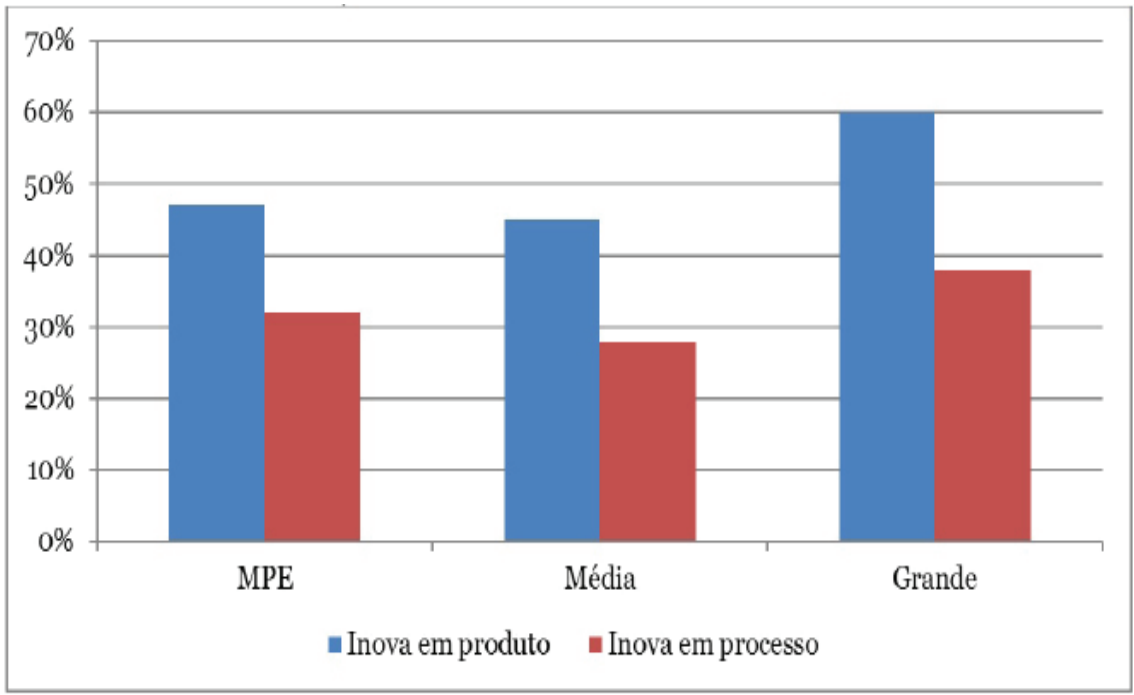

FONTE: pesquisa de campo, 2009. 
A Tabela 1 apresenta as estatísticas descritivas relacionadas ao percentual de empregados envolvidos em atividades de P\&D e a intensidade dos gastos em P\&D por tamanho da empresa. É interessante notar que, embora as grandes empresas apresentem uma taxa de inovação superior a das MPE, o percentual de empregados envolvidos em P\&D e a intensidade dos gastos em P\&D são menores. Enquanto as MPE possuem uma média de 19,4\% dos empregados envolvidos em P\&D, as grandes empresas possuem apenas 3,5\%. A média de gastos em P\&D das MPE é praticamente dois pontos percentuais superior a das grandes empresas. Esses números sugerem que os esforços realizados pelas MPE para inovar é consideravelmente superior ao das grandes empresas. O mesmo é verificado quando comparado com as médias empresas, sendo que a taxa de inovação é semelhante entre MPE e médias empresas.

TABELA 1. BRASIL: ESTATÍSTICAS DESCRITIVAS - 2009

\begin{tabular}{l|c|c|c|c}
\hline \multirow{2}{*}{$\begin{array}{c}\text { Tamanho da } \\
\text { empresa }\end{array}$} & \multicolumn{2}{|c|}{$\begin{array}{c}\text { Empregados envolvidos } \\
\text { em P\&D (\%) }\end{array}$} & \multicolumn{2}{c}{$\begin{array}{c}\text { Intensidade dos gastos em } \\
\text { P\&D (\% em relação a receita) }\end{array}$} \\
\cline { 2 - 5 } MPE & Média & Desvio Padrão & Média & Desvio Padrão \\
\hline Média & 19,4 & 22,0 & 5,8 & 8,3 \\
\hline Grande & 5,9 & 6,8 & 3,3 & 5,4 \\
\hline Média da amostra & 3,5 & 7,6 & 3,9 & 6,1 \\
\hline
\end{tabular}

FONTE: pesquisa de campo, 2009.

O padrão de distribuição das empresas entre os principais setores de atividade econômica presentes na amostra está representado na Figura 2. Mais da metade das empresas da amostra está relacionada ao setor de indústrias de transformação, sendo que para as médias empresas esse percentual ultrapassa a 70\%. As grandes empresas são maioria no setor de eletricidade e gás, onde é necessário ter grande escala de produção. Nos setores de atividades profissionais, científicas e técnicas e de informação e comunicação predominam as MPE. 
FIGURA 2. BRASIL: ATIVIDADE ECONÔMICA DAS EMPRESAS (\%) - 2009

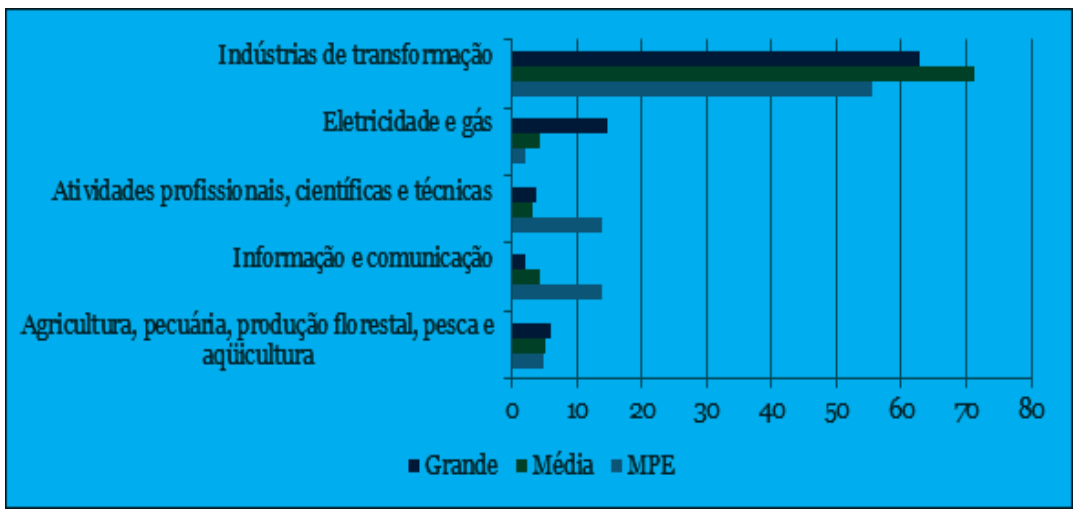

FONTE: pesquisa de campo, 2009.

As universidades e institutos de pesquisa foram apontados pelo conjunto das empresas como a terceira fonte mais importante de informação para sugerir novos projetos ou para concluir projetos já existentes, ficando atrás apenas da linha de produção da própria empresa ou de clientes. Para todas as categorias de tamanho de empresas, as universidades e institutos de pesquisa foram considerados mais importantes para concluir do que para sugerir novos projetos (ver Figura 3). Os resultados indicam que quanto menor a empresa, menos ela recorre às universidades e institutos de pesquisa para sugerir novos projetos. Entretanto, essa relação não se verifica quando se trata de concluir projetos. As MPE dão uma importância a essa fonte de informação semelhante às grandes empresas.

A interação com universidades e institutos de pesquisa ocorre por vários motivos. A Figura 4 apresenta as razões apontadas pelas empresas como sendo muito importante para a ocorrência dessa interação. Para simplificar a análise, destacamos apenas as razões apontadas pelos tamanhos extremos, ou seja, pelas grandes empresas e MPE.

Um dos resultados que se destacam é a diferença dada entre as grandes empresas e as MPE para a transferência de tecnologia das universidades. Enquanto mais de 60\% das grandes empresas apontaram essa razão como sendo muito importante para a colaboração, apenas 40\% das MPE indicaram o mesmo. A mesma diferença foi verificada para a questão da busca de conselhos tecnológicos. Apenas dois motivos para colaboração com universidades e institutos de pesquisa foram considerados mais importantes pelas MPE do que pelas grandes empresas: contato com estudantes e recebimento de ajuda no controle de qualidade. 
FIGURA 3. BRASIL: EMPRESAS POR TAMANHO QUE INDICARAM UNIVERSIDADES E INSTITUTOS DE PESQUISA COMO A FONTE MAIS IMPORTANTE DE INFORMAÇÃO PARA SUGERIR NOVOS PROJETOS OU PARA CONCLUIR PROJETOS EXISTENTES (\%) - 2009

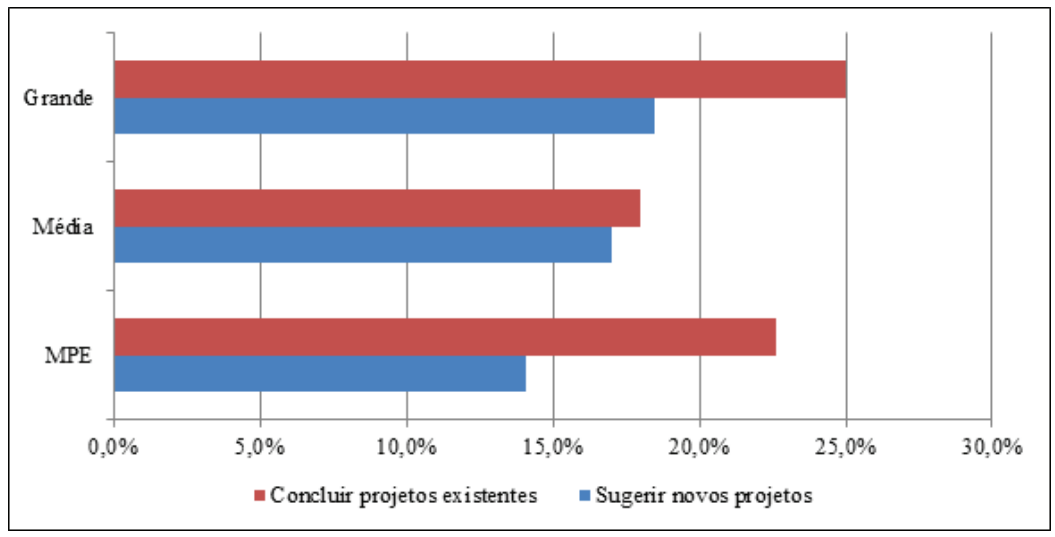

FONTE: pesquisa de campo, 2009.

FIGURA 4 . RAZÕES APONTADAS PELAS EMPRESAS, SEGUNDO O TAMANHO, COMO MUITO IMPORTANTES PARA A COLABORAÇÃO COM UNIVERSIDADES E INSTITUTOS DE PESQUISA (MPE E GRANDES EMPRESAS)

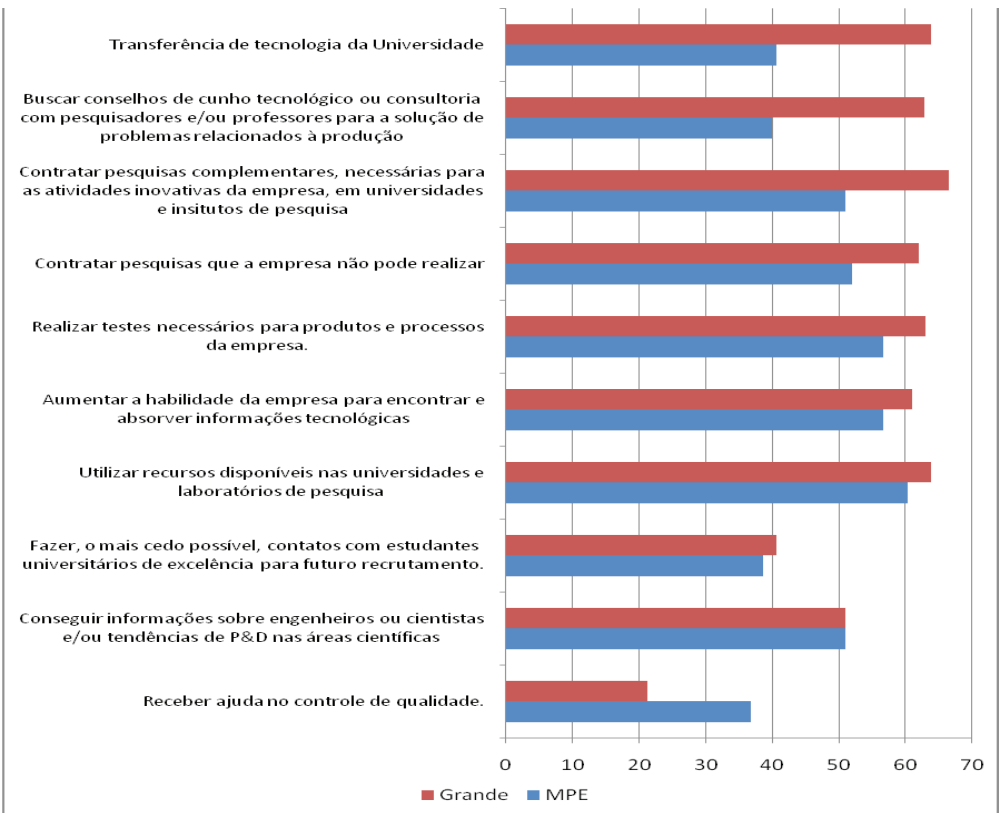

FONTE: pesquisa de campo, 2009. 
É interessante notar que as MPE e as grandes empresas dão praticamente a mesma importância a realização de testes e a utilização dos recursos laboratoriais disponíveis nas universidades e institutos de pesquisa como importante elemento para a colaboração.

\subsection{Modelo Econométrico}

Dando seguimento à análise dos dados fornecidos pelos questionários respondidos durante a pesquisa, esta seção tenta captar algumas evidências sobre a hipótese schumpeteriana da relação entre tamanho e propensão a inovar das empresas. Para tanto, é utilizado um modelo probit para estimar a probabilidade de que uma empresa tenha realizado algum tipo de inovação, de produto ou de processo, nos últimos três anos. O modelo pode ser sintetizado por:

$$
\operatorname{Pr}\left(y_{i}=1\right)=\Phi(x b)
$$

em que $y_{\mathrm{i}}$ é a variável dependente binária que assume valor 1 se a firma realizou alguma inovação no período e valor zero em caso contrário; $\Phi($.$) é$ a função de distribuição acumulada de uma variável aleatória padronizada, com média o e variância $1 ; x$ é o conjunto de variáveis explicativas e $b$ o vetor de coeficientes a ser estimado.

Como variáveis explicativas, o presente trabalho utiliza um conjunto de dummies para captar o tamanho da empresa (MPE, média e grande empresa) representando a hipótese da relação entre o tamanho e a propensão a inovar, uma dummy de valor 1 para identificar as firmas do setor de indústrias de transformação (que possuem a maior representatividade na amostra selecionada) e a porcentagem de trabalhadores ocupados em atividades de pesquisa e desenvolvimento dentro de cada empresa.

Contudo, o modelo probit original supõe a existência de variância constante na variável dependente $y_{\mathrm{i}}$, o que pode não ser verdadeiro na prática. Este problema pode ser testado e corrigido usando a versão própria para heterocedasticidade do estimador probit, tal como propõe, por exemplo, Greene (2003) e Cameron e Trivedi (2009), generalizando a função $\Phi($. para o caso em que a variância é uma função de um conjunto $z$ de variáveis explicativas. Ou seja:

$$
\sigma_{\mathrm{i}}^{2}=\exp \left(z_{\mathrm{i}}^{\prime} \delta\right)
$$

em que $z_{\mathrm{i}}$ é uma variável que explica a heterocedasticidade e $\delta$ capta a relação desta variável com a variância. Se $\delta$ é estatisticamente igual a zero, pode-se aceitar a hipótese nula de ausência de heterocedasticidade causada por $z_{\mathrm{i}}$. 
Para a presente análise, consideramos a hipótese de que a heterocedasticidade é uma função da intensidade de investimento em P\&D da firma. Vários estudos tem mostrado a substancial variação da intensidade de investimento em P\&D, tanto entre setores industriais, quanto entre tamanhos de firmas (Macedo e Albuquerque, 1998; Façanha e Resende, 2004).

Os coeficientes estimados dos modelos para a probabilidade de se observar uma firma inovadora em produto ou em processo podem ser visualizados nas Tabelas 2 e 3, respectivamente. Os resultados dos testes de significância de $\delta$ confirmam a presença de heterocedasticidade causada pela intensidade do investimento em P\&D no modelo de inovação de produto, mas não quando se estima para a inovação em processo. Isso indica que o mais adequado seria a utilização do modelo probit tradicional para o caso da atividade de inovação em processo. Contudo, como os resultados dos dois modelos, tanto em termos de significância individual dos coeficientes, quanto a significância global, são semelhantes, os modelos tradicionais são expostos nos anexos do artigo.

TABELA 2. BRASIL: RESULTADO DA APLICAÇÃO DO MODELO PROBIT HETEROSCEDÁSTICO, PROBABILIDADE DE INOVAR EM PRODUTO - 2009

\begin{tabular}{l|c|c|c}
\hline Média Empresa & $(1)$ & $(2)$ & $(3)$ \\
\hline Grande Empresa & $\begin{array}{c}0.2090 \\
(0.3421)\end{array}$ & $\begin{array}{c}0.0576 \\
(0.3001)\end{array}$ & $\begin{array}{c}0.2309 \\
(0.3440)\end{array}$ \\
\hline Indústria Transformação & $\begin{array}{c}1.0124^{*} \\
(0.3505)\end{array}$ & $\begin{array}{c}0.8260^{*} \\
(0.2939)\end{array}$ & $\begin{array}{c}1.0099^{*} \\
(0.3487)\end{array}$ \\
\hline Empregados em P\&D & 0.3116 & 0.2882 & \\
\hline Constante & $(0.2644)$ & $(0.2577)$ & 10.549 \\
& $(10.993$ & & $(11.342)$ \\
\hline Observações & $-0.8948^{* *}$ & $-0.6696^{* *}$ & $-0.7027^{* *}$ \\
Wald chi2 & $(0.3740)$ & $(0.2921)$ & $(0.3270)$ \\
\hline Prob>chi2 & 296 & 296 & 296 \\
\hline Teste para Sigma $=0$ & 1.132 .689 & 1.068 .957 & 1.051 .652 \\
\hline Prob<chi2 & .023126 & .013529 & .014649 \\
\hline NOTA & 39.669 & 29.948 & 30.473 \\
\hline
\end{tabular}

NOTA: ${ }^{* * *} \mathrm{p}<0.10,{ }^{* *} \mathrm{p}<0.05,{ }^{*} \mathrm{p}<0.01$. Desvios padrão entre parênteses.

FONTE: resultados obtidos a partir de dados da Pesquisa de Campo, 2009. 
TABELA 3. BRASIL: RESULTADO DA APLICAÇÃO DO MODELO PROBIT HETEROSCEDÁSTICO, PROBABILIDADE DE INOVAR EM PROCESSO - 2009

\begin{tabular}{|c|c|c|c|}
\hline & (1) & (2) & (3) \\
\hline Média Empresa & $\begin{array}{c}0.1312 \\
(0.1877)\end{array}$ & $\begin{array}{c}0.0278 \\
(0.1787)\end{array}$ & $\begin{array}{c}0.1276 \\
(0.1869)\end{array}$ \\
\hline Grande Empresa & $\begin{array}{l}0.3799^{* *} \\
(0.1898)\end{array}$ & $\begin{array}{c}0.2599 \\
(0.1746)\end{array}$ & $\begin{array}{l}0.3842^{* *} \\
(0.1879)\end{array}$ \\
\hline Indústria Transformação & $\begin{array}{l}-0.0885 \\
(0.1479)\end{array}$ & $\begin{array}{l}-0.1448 \\
(0.1478)\end{array}$ & \\
\hline Empregados em P\&D & $\begin{array}{c}0.7517 \\
(0.4790)\end{array}$ & & $\begin{array}{r}0.8189^{* * *} \\
(0.4679)\end{array}$ \\
\hline Constante & $\begin{array}{l}-0.6424^{*} \\
(0.1887)\end{array}$ & $\begin{array}{l}-0.4680^{*} \\
(0.1499)\end{array}$ & $\begin{array}{l}-0.7009^{*} \\
(0.1741)\end{array}$ \\
\hline $\begin{array}{l}\text { Observações } \\
\text { Wald chi2 }\end{array}$ & $\begin{array}{l}296.000 \\
5.39696\end{array}$ & $\begin{array}{l}296.000 \\
3.283277\end{array}$ & $\begin{array}{c}296.000 \\
5.394502\end{array}$ \\
\hline Prob $>$ chi2 & .24894 & .34998 & .14509 \\
\hline Teste para Sigma $=0$ & 1.1486 & .54516 & 1.3256 \\
\hline Prob $<$ chi2 & .28385 & .4603 & 24959 \\
\hline
\end{tabular}

NOTA: ${ }^{* * *} \mathrm{p}<0.10,{ }^{* *} \mathrm{p}<0.05,{ }^{*} \mathrm{p}<0.01$. Desvios padrão entre parênteses.

FONTE: resultados obtidos a partir de dados da Pesquisa de Campo, 2009.

De modo geral, os modelos estimados mostram uma influência do tamanho da empresa, medido em termos do número de empregados, tanto sobre a propensão a inovar em produtos como na inovação de processos. Tomando as MPE como referência, os sinais positivos e significativos da dummy que representa as grandes empresas mostram uma diferença relevante em termos de propensão a inovar nos dois modelos estimados (o mesmo se observa nos modelos probit tradicionais em anexo, confirmando estes resultados).

Contudo, o modelo de inovação em processo não se mostra significativo globalmente, o que permite inferir que a hipótese de relação entre o tamanho da empresa e a atividade de inovação é válida apenas, considerando a amostra empregada, para a inovação em produto.

Com relação às demais variáveis de controle, a amostra de empresas que interagiram com universidades e institutos de pesquisa não revela diferença significativa na propensão a inovar entre as firmas do setor de indústria de transformação e os demais setores. O percentual da mão de obra envolvida nas atividades de P\&D também não é relevante no modelo de inovação de produto, sendo apenas nos modelos estimados para a inovação de processo. 


\section{Conclusão}

A hipótese schumpeteriana de que empresas maiores tendem a ser mais inovadoras que as menores foi testada a partir de um conjunto de dados de empresas que interagem com universidades e institutos de pesquisa.

Os dados analisados sugerem que os esforços realizados pelas MPE para inovar são consideravelmente maiores que os das médias e grandes empresas.

Os resultados do exercício econométrico sugerem que o porte da empresa tem uma relação significativa e positiva com a propensão a inovar em produto. O mesmo não se verifica com a mesma consistência para inovações de processo. Portanto, a denominada hipótese schumpeteriana é confirmada apenas em parte, pois depende do tipo de inovação em questão.

Ao realizar o controle pelo tamanho da empresa e pelo percentual de empregados em atividades de $\mathrm{P} \& \mathrm{D}$, a amostra não revela diferença significativa na propensão a inovar entre as firmas do setor de indústria de transformação e os demais setores.

De maneira geral, os resultados sugerem que existem diferenças significativas de padrão de inovação em empresas de diferentes portes e que políticas voltadas ao estímulo da inovação em MPE podem ser justificadas pelo grande esforço que as mesmas precisam empreender para poder inovar.

Contudo, lembramos que os resultados aqui apresentados são limitados pela amostra em questão e pela ausência de um grupo de controle. Estudos mais amplos utilizando os dados da PINTEC ajudariam a relativizar com maior qualidade o padrão de inovação entre empresas de diferentes tamanhos. 


\section{Referências bibliográficas}

ACS, Z; AUDRETSCH, D. (1987).” Innovation, Market Structure, and Firm Size.” The Review of Economics and Statistics, 69(4); p. 567-574.

CAMERON, A. C.; TRIVEDIP, K. (2009). Microeconometrics using Stata. Stata Press.

COHEN, W.; LEVIN, R. (1989). "Empirical studies of innovation and market structure." Handbook of Industrial Economics, 2.

FAÇANHA RESENDE. (2004). "R\&D Intensity in the Brazilian Industry: Some Distributional Regularities”. Revista Brasileira de Inovação, 3(2); p. 309-321.

FREEMAN, C.; SOETE, L. (2008). A Economia da Inovação Industrial. Campinas: Editora Unicamp. Coleção Clássicos da Inovação.

GREENE, W.H. (2003). Econometrics analysis. New Jersey: Prentice-Hall.

KANNEBLEY JR, S.; PORTO, G. S.; PAZZELO, E. T. (2004). "Inovação na indústria brasileira: uma análise exploratória a partir da PINTEC.” Revista Brasileira de Inovação, 3(1); p. 87-128.

LEVIN, R.; KLEVORICK, A.; NELSON, R.; WINTER, S. (1987). “Appropriating the returns from industrial research and development.” Brookings Papers on Economic Activity, 3; p. 783-831.

MACEDO, P. B. \& ALBUQUERQUE, E. M. (1999). "P\&D e Tamanho da Empresa: Evidência Empírica sobre a Indústria Brasileira.” Estudos Econômicos, 29(3); p. 343-365.

MAIRESSE, J.; MOHNEN, P. (2010). "Using innovation surveys for econometric analysis". In: HALL, B. H.; ROSENBERG, N. Handbook of the Economics of Innovation, 3; p. 1129-1156. Burlington: Academic Press.

SCHUMPETER, J. (1942). “Capitalismo, Socialismo e Democracia.” URL [on-line]: http://www.ordemlivre.org/files/schumpeter-csd.pdf. Acesso em: jun. de 2011. 


\section{Anexos}

TABELAA1. RESULTADODAAPLICAÇÃODOMODELO PROBIT, PROBABILIDADE DE INOVAR EM PRODUTO, BRASIL - 2009

\begin{tabular}{l|c|c|c}
\hline & $\mathbf{( 1 )}$ & $\mathbf{( 2 )}$ & $\mathbf{( 3 )}$ \\
\hline Média Empresa & -0.0389 & -0.0861 & -0.0308 \\
\hline Grande Empresa & $(0.1941)$ & $(0.1818)$ & $(0.1937)$ \\
\hline & $0.3780^{* * *}$ & $0.3196^{* * * *}$ & $0.3740^{* * * *}$ \\
\hline Indústria Transformação & $(0.1969)$ & $(0.1783)$ & $(0.1966)$ \\
\hline & 0.1109 & 0.0899 & \\
\hline Empregados em P\&D & $(0.1556)$ & $(0.1530)$ & \\
\hline & 0.3730 & & 0.3010 \\
\hline Constante & $(0.5366)$ & & $(0.5276)$ \\
\hline Observações & -0.1965 & -0.1120 & $(0.1614)$ \\
\hline Wald chi2 & $(0.1932)$ & $(0.1513)$ & 296.000 \\
\hline Prob>chi2 & 296.000 & 296.000 & 5.869514 \\
\hline
\end{tabular}

NOTA: ${ }^{* * *} \mathrm{p}<0.10,{ }^{* *} \mathrm{p}<0.05,{ }^{*} \mathrm{p}<0.01$. Desvios padrão entre parênteses.

FONTE: resultados obtidos a partir de dados da Pesquisa de Campo, 2009.

TABELAA2. RESULTADODAAPLICAÇÃODOMODELO PROBIT,PROBABILIDADE DE INOVAR EM PROCESSO, BRASIL - 2009

\begin{tabular}{l|c|c|c}
\hline & (1) & (2) & (3) \\
\hline Média Empresa & 0.1237 & 0.0208 & 0.1180 \\
\hline Grande Empresa & $(0.2110)$ & $(0.1946)$ & $(0.2108)$ \\
\hline & $0.4146^{* *}$ & 0.2861 & $0.4198^{* *}$ \\
\hline Indústria Transformação & $(0.2083)$ & $(0.1853)$ & $(0.2086)$ \\
\hline & -0.1101 & -0.1559 & \\
\hline Empregados em P\&D & $(0.1622)$ & $(0.1595)$ & \\
\hline & 0.7795 & & 0.8542 \\
\hline Constante & $(0.5566)$ & & $(0.5493)$ \\
\hline Observações & $-0.6893^{*}$ & $-0.5051^{*}$ & $-0.7652^{*}$ \\
\hline Wald chi2 & $(0.2054)$ & $(0.1569)$ & $(0.1780)$ \\
\hline Prob>chi2 & 296.000 & 296.000 & 296.000 \\
\hline
\end{tabular}

NOTA: ${ }^{* * *} \mathrm{p}<0.10,{ }^{* *} \mathrm{p}<0.05,{ }^{*} \mathrm{p}<0.01$. Desvios padrão entre parênteses.

FONTE: resultados obtidos a partir de dados da Pesquisa de Campo, 2009. 